\title{
Cartografía social: construyendo territorio a partir de los activos comunitarios en salud *
}

\author{
Diana Paola Betancurth Loaiza \\ Docente Universidad de Caldas, Manizales - Colombia \\ diana.betancurth@ucaldas.edu.co (D) https://orcid.org/0000-0001-7620-2336
}

Consuelo Vélez Álvarez

Docente Universidad de Caldas, Manizales - Colombia

consuelo.velez@ucaldas.edu.co $\quad$ (D) https://orcid.org/0000-000 l-7620-2336

\author{
Natalia Sánchez Palacio \\ Universidad de Caldas, Manizales, Colombia \\ nataliasp.palacio@gmail.com (D) https://orcid.org/0000-0002-3405-5/44
}

RES U M N

Existe la necesidad de identificar herramientas participativas que permitan explorar los activos para la salud a partir del reconocimiento del territorio por parte de la comunidad. Objetivo: evidenciar la utilización de la cartografía social como método para el estudio de los activos en salud a nivel comunitario. Metodología: se presenta la experiencia de la cartografía social, basada en el análisis de la categoría "diseño metodológico" del estudio base, con lo cual se consolida el sentido de pertenencia a partir de representaciones gráficas de la realidad existente en la comunidad. Resultados: las orientaciones detalladas sobre cómo realizar un estudio para conocer los activos comunitarios a través de la cartografía social son mostradas a través de la experiencia de las investigadoras en campo. Se desarrollaron cinco etapas: conocimiento del territorio, acercamiento a la comunidad, utilización de herramientas de indagación comunitaria, proceso de mapeo y establecimiento de acuerdos. Conclusión: la identificación de los activos comunitarios hace posible que la comunidad reconozca sus fortalezas personales, familiares y sociales, reflexionen y analicen su realidad, reconociendo aquellos elementos en los que se desarrolla su vida cotidiana, por tanto, podría afirmarse que la cartografía social aporta para la elaboración de rutas de vida a nivel comunitario.

Palabras clave

Cartografía Social, atención primaria en salud, comunidad, participación de la comunidad, mapas (Fuente: DeCS, BIREME)

\section{Social cartography: building territory from community assets in health}

A B S T R A C T

There is a need to identify participatory tools to explore health assets based on community recognition of the territory. Objective: to highlight the use of social mapping as a method for the study of health assets at the community level. Methodology: the experience of social mapping is presented, based on the analysis of the "methodological design" category of the base study, which consolidates the sense of belonging based on graphic representations of the existing reality in the community. Results: Detailed guidelines on how to

\footnotetext{
* http://dx.doi.org/ Este es un artículo Open Access bajo la licencia BY-NC-SA (http://creativecommons.org/licenses/by-nc-sa/4.0/)

Cómo citar este artículo: BETANCURTH LOAIZA, Diana Paola;VÉLEZ ÁLVAREZ, Consuelo y SÁNCHEZ PALACIO, Natalia. Cartografía social: construyendo territorio a partir de los activos comunitarios en salud. En: Entramado. Enero - Junio, 2020 vol. 16, no. I, p. I38-151 
conduct a study to learn about community assets through social mapping are shown through the experience of the field researchers. Five stages were developed: knowledge of the territory, approach to the community, use of community research tools, mapping process and establishment of agreements. Conclusion: the identification of community assets makes it possible for the community to recognize its personal, family and social strengths, to reflect on and analyze its reality, recognizing those elements in which its daily life is developed. Therefore, it could be said that social mapping contributes to the elaboration of life routes at the community level.

KEYWORDS Social Cartography, primary health care, community, community participation, maps (Source: DeCS, BIREME)

\section{Mapeamento social: construindo território a partir de ativos de saúde da comunidade}

É necessário identificar ferramentas participativas que permitam a exploração de ativos de saúde com base no reconhecimento do território pela comunidade. Objetivo: demonstrar o uso da cartografia social como método de estudo dos ativos de saúde em nível comunitário. Metodologia: é apresentada a experiência da cartografia social, com base na análise da categoria "desenho metodológico" do estudo de base, que consolida o sentido de pertencimento a partir das representações gráficas da realidade existente na comunidade. Resultados: Orientações detalhadas sobre como conduzir um estudo para aprender sobre os ativos da comunidade através do mapeamento social são mostradas através da experiência de pesquisadores da área. Foram desenvolvidas cinco etapas: conhecimento do território, abordagem à comunidade, uso de ferramentas de investigação da comunidade, processo de mapeamento e estabelecimento de acordos. Conclusão: a identificação dos ativos da comunidade possibilita que a comunidade reconheça suas forças pessoais, familiares e sociais, reflita e analise sua realidade, reconhecendo os elementos em que seu cotidiano é desenvolvido, portanto, pode-se afirmar que o mapeamento social fornece para o desenvolvimento de rotas de vida em nível comunitário.

Palavras-chave Mapeamento social, atenção primária à saúde, comunidade, participação da comunidade, mapas (Fonte: DeCS, BIREME)

\section{Introducción}

La elaboración de mapas proviene de una larga tradición de trabajo participativo. Su realización -como una imagen estática- sin la intervención directa de la comunidad, se configura como un ejercicio que oculta las realidades territoriales concretas que a la vez suelen ser complejas. Para Ganter, Sandoval, García y De la Fuente (2015), los diagnósticos participativos son una "fotografía" realizada por la comunidad de sus relaciones sociales y de la manera como interactúa con su entorno, según las temáticas y elementos más relevantes en su diario vivir.

En este sentido, la realización de procesos de investigación participativa, busca en esencia generar transformación de realidades de aquellos actores que hasta el momento han sido sujetos pasivos en la interacción con los investigadores, para que a partir de la utilización de nuevas metodologías, técnicas y estrategias se conviertan en protagonistas de la generación de nuevo conocimiento (Yepes y Marín, 2018).
La cartografía en la investigación social parte de la premisa: "quien vive en el territorio es quien lo conoce", por tanto, se debe recurrir a los habitantes para poderlo comprender (Pájaro y Tello, 20l4). Barragán y Amador (20l4), la describen como una metodología que posibilita el abordaje no solo de problemas sociales, sino también de la manera como se integran los diferentes actores en los procesos de co-construcción social del conocimiento, intercambio colectivo de saberes y adicionalmente, en la identificación de sus recursos para la transformación de sus realidades. Estos recursos para el caso de los activos comunitarios se dan en sentido positivo, reconociendo de manera explícita cuáles son y lo que aportan al desarrollo comunitario.

Para la salud pública el modelo de activos aporta una nueva mirada a la salud, en la que las personas y comunidades analizan su contexto e identifican en él todos aquellos elementos que pueden mejorar de manera integral su salud y además, producen bienestar no solo individual, sino también comunitario (Iglesias et al., 20l8). 
El enfoque de los activos comunitarios y los activos para la salud busca retomar las acciones de salud pública para un territorio a partir de los recursos reconocidos por la comunidad, afianzados desde la promoción de la salud, motivándola para que reoriente la mirada de salud desde aquellos factores y recursos que aportan a su bienestar y a la búsqueda de una mayor equidad involucrando a todos los integrantes de las colectividades (Cofiño et al., 20l6).

La realización de proyectos de investigación en salud utilizando el método de cartografía social, asume el lograr que las comunidades reconozcan su territorio como espacio vivo, con sentido de vida y para el desarrollo de su vida y por lo tanto, permite documentar la evidencia del empoderamiento comunitario para el desarrollo social integral (Montes et al., 2015-2016).

El objetivo del presente artículo es evidenciar la utilización de la cartografía social como método para el estudio de los activos en salud a nivel comunitario.

\section{Marco teórico}

\section{I. Cartografía social}

La cartografía social, mapa social o sociograma, es considerada como una opción metodológica que permite realizar una aproximación de una concepción sobre determinado territorio en permanente cambio, a la vez que permite consolidar sentido de pertenencia a partir de representaciones gráficas de la realidad existente en la comunidad (Barragán y Amador, 2014; Uribe, Donoso y Ramírez, 2017).

Así, en la cartografía social se asume el mapa como una representación gráfica de aquellos fenómenos presentes en una comunidad -se concibe como un medio, no como un fin-, en el cual se esquematiza la realidad existente y las relaciones territoriales a través de signos, símbolos y palabras que se identifican por medio de convenciones y que tienen significados compartidos y proyecciones colectivas (Barragán y Amador, 2014).

Para este proceso es necesario tener en cuenta las características individuales, sociales y políticas de los colectivos pues esto determinará la manera en que se llevará a cabo la cartografía a fin de garantizar todas las condiciones necesarias para que los participantes puedan reflexionar y exponer las percepciones, representaciones e interpretaciones de su realidad. Por lo anterior, la cartografía social se sitúa en el enfoque comprensivo-crítico que contribuye a develar aquellos aspectos simbólicos que les permite a los sujetos interpretar su mundo y transformarlo
Por lo anterior, para reconocer la cartografía social como una opción metodológica es necesario partir desde una perspectiva territorial que permita el fortalecimiento comunitario desde la creación de autorrepresentaciones, autorreconocimiento y análisis de la información (Barragán, 2019).

\section{I.I. Tipos de mapeos colectivos}

Para la realización adecuada de la cartografía social es necesario diferenciarla de un mapa geográfico, este último tiene aproximaciones objetivas del territorio, y el primero realiza una representación gráfica de una realidad social con la elaboración de relatos colectivos (Uribe et al., 20I7).

En función de lo anterior, Barragán (2016) propone tres tipos de mapas en el proceso de cartografía social que permiten priorizar reflexiones colectivas, reconocer los territorios, relaciones, necesidades, saberes y oportunidades de transformación en los actores sociales, por lo que es necesario tenerlos en cuenta antes de iniciar el ejercicio con la comunidad:

- Mapa ecosistémico-poblacional (relaciones territoriales). Su propósito es hacer emerger las relaciones territoriales partiendo de los vínculos y rupturas entre población y naturaleza, más allá de las delimitaciones político-administrativas.

- Mapa temporal-social (de pasado, de presente, de futuro). A través de este se reconocen los acontecimientos que se encuentran en la memoria de una comunidad para comprender el presente y representar el futuro.

- Mapa temático (con problemáticas y planificaciones concretas). Permite comprender fortalezas y debilidades de una comunidad, y convertirlas en situaciones problemáticas y en posibilidades de transformación.

\subsection{Activos comunitarios en salud}

Pensar en los activos es una forma de ver las acciones en salud como aquello que hace que las personas, las familias $y$ las comunidades controlen y mejoren su salud, cuestiones relacionadas con la salutogénesis (Antonovsky, 1987a; Scales, 1999) y el origen de la salud, que se entiende en términos de un continuo -bienestar/salud y malestar/enfermedad-, que reconoce los recursos de las personas para que vivan más felices, más allá de una visión de salud y enfermedad que se excluyen mutuamente.

El enfoque de la salud pública centrado en un modelo biomédico -prevención- está dando un cambio hacia la salud pública positiva. La tendencia clásica de la orientación hacia los factores de riesgo o generación de barreras para 
la enfermedad es lo que ha predominado. Es por ello que se intenta reorientar hacia una forma de ver las acciones en salud, focalizando en lo biopsicosocial, sustentándose en las oportunidades para conservar la salud o mejorarla, un modelo muy ligado al origen y desarrollo de la Atención Primaria en Salud -APS- (De la Revilla, 1993).

La óptica no patogénica, conocida también como perspectiva salutogénica de Antonovsky (1987b), pone énfasis en el origen de la salud y no en el origen de la enfermedad. Se trata de un proceso diferente al diagnóstico o la intervención sobre las falencias de individuos y comunidades; consiste en la identificación de activos. Un activo se define como cualquier factor o recurso que potencie las capacidades de los individuos, de las comunidades y poblaciones (Antonovsky, I987a; Morgan y Ziglio, 2007; Morgan, Davies y Ziglio, 2010).

La exploración de procesos salutogénicos, a través de la construcción de un mapa de activos en salud -identifica fortalezas- (Antonovsky, 1987a; Scales, 1999), permite descubrir los recursos individuales, colectivos y ambientales ya existentes en el territorio. Implica reconocer los talentos, habilidades, intereses y experiencias de la comunidad, y conocimientos valiosos en la construcción de alternativas para la atención en salud, cuestiones que van más allá de una lista de problemas o necesidades usadas para la asignación de recursos. Es una alternativa en el proceso de apoderamiento' (Scales, 1999), participación, capacitación y responsabilidad no solo individual sino también de todos los actores sociales de la comunidad. Bajo este modelo la investigación en salud debe definir y describir las rutas, las causas y factores que pueden complementar el conocimiento de los profesionales de salud sobre cómo gestionar la salud y prevenir la enfermedad.

Los conceptos fundamentales que Antonovsky (1987a) desarrolla con esta teoría salutogénica son los Recursos Generales de Resistencia (GRRs) y el Sentido de Coherencia (SOC). Los GRRs que sustentan el comportamiento del individuo y proceden de su propio yo, de su contexto sociocultural y del entorno físico y natural, son aquellos recursos que hacen que la vida pueda percibirse de una forma coherente, estructurada y comprensible (el dinero, el conocimiento, la experiencia, la autoestima, los hábitos saludables, el compromiso, el apoyo social, el capital cultural, la inteligencia, las tradiciones y la visión de la vida). No obstante, más allá de poseer estos recursos, lo importante es tener la capacidad para utilizarlos, es decir, poseer lo que Antonovsky llamó SOC (Sense of Coherence), convirtiéndose dicho concepto en la clave de su teoría.

De esta manera, el modelo de activos en salud aporta una perspectiva de salud que incentiva la participación comunitaria en el contexto, centrado en aquello que potencie la equidad en salud, fortalezca sus decisiones sobre sus problemáticas, desde un enfoque alternativo que se aleja de la medicalización, el consumismo y la dependencia del sistema sanitario o de los servicios sociales, al tomar conciencia del trabajo comunitario que aprovecha al máximo sus talentos y habilidades (Cofiño et al., 20l6).

Estas iniciativas participativas en salud a nivel internacional aparecen en la Declaración de Alma-Ata (Organización Mundial de la Salud, 1978), donde se afirma que el pueblo tiene derecho y deber de participar tanto individual como colectivamente en la planificación de su atención de salud, e incluso fomenta la autorresponsabilidad y la participación de la comunidad en la organización, el control y funcionamiento de la APS en función del aprovechamiento de recursos locales, mediante la educación y el incentivo a la participación comunitaria. De igual modo, la Carta de Ottawa para la Promoción de la Salud (Organización Mundial de la Salud, 1986) reorienta los servicios sanitarios hacia una responsabilidad conjunta de personas, grupos comunitarios y gobiernos, desde un enfoque más amplio que incluya las necesidades culturales y sea sensible ante las realidades sociales, políticas y económicas de las comunidades.

Es preciso aplicar en la práctica metodologías que permitan a los profesionales de la salud fortalecer su formación, a la vez que potencien sus capacidades para colaborar, identificar recursos, elementos culturales y ambientales ya existentes en el contexto para conectar mejor sus saberes con los de la comunidad y lograr responder de manera concreta a las necesidades de las personas.

\section{Metodología}

Se llevó a cabo un estudio a través de cartografía social, la cual tiene sus raíces en la Investigación Acción Participación (IAP), dado el carácter dinámico de la construcción del mapa y el diálogo emergente en el proceso del trabajo colectivo en torno a lo que allí se representa (Barragán, 2016). Subyace de un proceso netamente subjetivo y cualitativo, por un lado, debido a las estrategias utilizadas para la recolección de la información que se deriva principalmente de las vivencias de los actores del territorio y del equipo investigador, y por el otro, dado el análisis y los significados que se le atribuyen a los resultados por parte de los investigadores y lectores (Uribe et al., 2017).

Para esta investigación se tomaron elementos centrales de las cartografías:

- Cartografía de sentidos, percepciones y territorios:con la cual se identificaron sonidos, olores, sentimientos, 
sabores, vivencias y todos aquellos elementos que resultaran significativos para los participantes.

- Cartografía de activos en salud: en esta, se plasmaron los recursos que los participantes reconocieron del barrio, siendo estos importantes para su salud individual y comunitaria.

- Cartografía de recorridos urbanos y cartografía de paisajes: para la realización de estos tipos de cartografía se realizó un recorrido colectivo de mapeo en movimiento y captura de fotografías del barrio, con el fin de realizar la recolección de insumos visuales y sensoriales que permitieran complementar la identificación de activos en salud.

La experiencia del proceso de la cartografía social -el cómo se hace-, que se presenta en este manuscrito, se realizó entre mayo de 2018 y mayo de 2019 , en cuatro barrios del departamento de Caldas (Colombia), que tienen la particularidad de ser territorios que han sido identificados de riesgo, (con un riesgo acumulado entre 3,29 y 3,89 significa un riesgo medio-bajo ${ }^{2}$ ). Este proceso se articuló con las acciones de APS de los municipios y se centró en la comunidad como unidad de análisis, con una mayor participación de líderes y lideresas comunitarios con quienes se planeó y coordinó el contenido y la logística de las actividades, como una muestra de respeto a la gobernabilidad de los territorios que representan.
Se abordaron diferentes aspectos como ubicación geográfica, determinantes sociales, percepciones de su entorno, creencias, actitudes, historicidad, antecedentes de acompañamiento comunitario y economía local, los cuales nutrieron información para la representación de los activos como fenómeno central para la investigación.

Según las dinámicas de los territorios se fueron adaptando diferentes maneras de acercamiento a la comunidad. Entre las técnicas utilizadas estuvieron las entrevistas, la observación participante y otras actividades como talleres, mingas, visitas informales, recorridos en campo y jornadas recreativas, todo esto paralelo al proceso de mapeo colectivo, en el cual participaron adultos mayores, adultos, jóvenes y niños. En promedio, participaron 50 personas del tejido social, una persona con formación técnica y seis profesionales. En algunos barrios estas maneras fueron emergentes dado el proceso de reinterpretación continua de las dinámicas territoriales.

La información fue codificada en Atlas.ti 7.0 - licenciado por la Universidad de Caldas-. Para el análisis se exportó una de las nueve categorías obtenidas en el proceso ${ }^{3}$ (metodología). La información se ordenó y con la experiencia del trabajo de campo se establecieron las etapas presentadas en la Figura I.

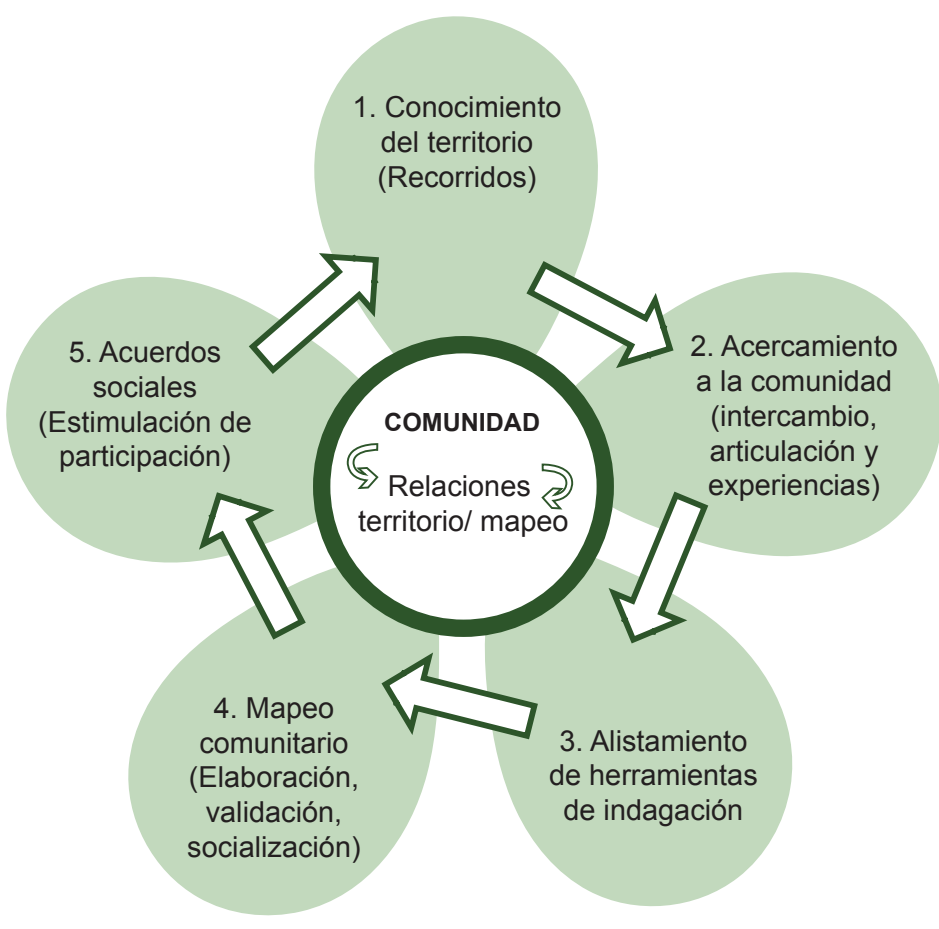

Figura I. Etapas de la cartografía social para la identificación de activos comunitarios en salud. Fuente: Elaboración propia. 
Para la realización de la investigación, las autoras tuvieron en cuenta como criterios de evaluación de la calidad (Calderón, 2002) la adecuación epistemológica, en tanto la investigación contribuye a describir la experiencia de la cartografía y con ello brindar el insumo para una fase siguiente de significados subjetivos frente a los activos del territorio; relevancia, dado que permite reconocer el territorio con la comunidad, siendo esto una base para avanzar a la apropiación y reflexión del mismo, lo que conlleva finalmente a la formulación de políticas públicas coherentes con la realidad territorial; validez, la investigación estuvo direccionada hacia la búsqueda de explicaciones en profundidad de los activos comunitarios en salud, lo que derivó en la construcción del presente artículo que da cuenta del rigor metodológico empleado para el cumplimiento de los objetivos; reflexividad, ha sido la posibilidad de interpretaciones desde el inicio del estudio por parte de los investigadores teniendo en cuenta sus puntos de vista y el reconocimiento de los sujetos participantes.

\section{I. Consideraciones éticas}

Para el desarrollo del trabajo con la comunidad se hizo necesario contar con el consentimiento informado de los integrantes de los diferentes grupos de actores, por tanto, antes del inicio de cada sesión se procedió al diligenciamiento del mismo. Lo anterior, en cumplimiento de los parámetros expresados en la Declaración de Helsinki y en la Resolución 08430 de 1993 para el territorio colombiano. El proyecto de investigación fue aprobado por el comité de ética de la Universidad de Caldas con el código CBCS-065.

\section{Resultados}

Los resultados son presentados a la luz del objetivo del presente artículo, evidenciar la utilización de la cartografía social como método para el estudio de los activos en salud a nivel comunitario y el cual deriva de la experiencia de las autoras con el mismo proceso, cuya reflexión teórica se evidencia en la discusión como aporte metodológico a la praxis en el marco de la salud pública.

Los activos comunitarios presuponen una revitalización de los territorios desde miradas esperanzadoras y de salud positiva, alejadas del modelo de riesgo o de revictimización, como se observa en la Figura 2, más allá de un espacio geográfico, para la salud pública el proceso cartográfico devela recursos como: relaciones sociales,

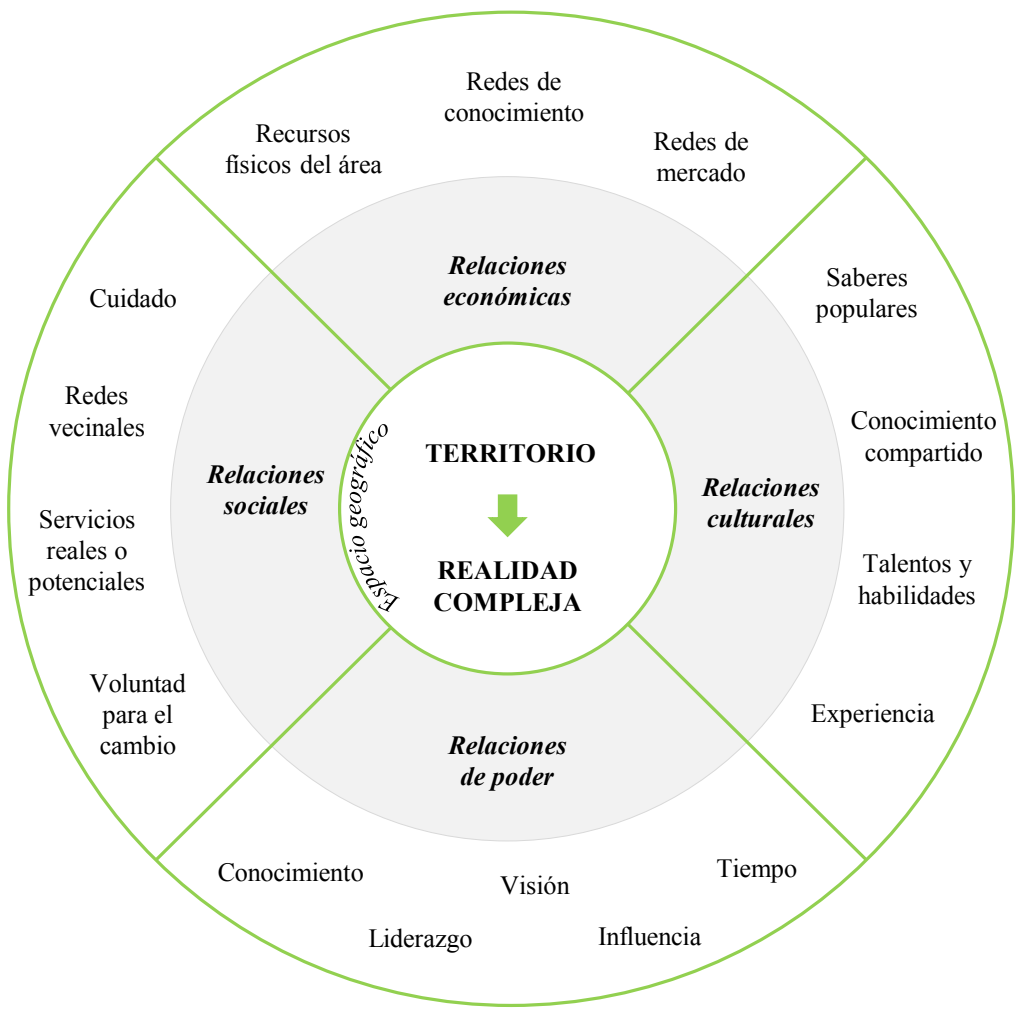

Figura 2. Relación cartografía social y activos comunitarios en salud. Fuente: Elaboración propia 
de poder, culturales y económicas que son claves para el direccionamiento de programas, proyectos e intervenciones en promoción de la salud, desde una mirada colaborativa cuyo punto de partida es el patrimonio existente en las comunidades.

A continuación se describen cinco etapas del proceso investigativo, las cuales configuraron la ruta - el medio-, que a la vez se convierte en proceso reflexivo para llegar al análisis de los activos en relación con el territorio.

\section{I. Conocimiento del territorio}

Previo a iniciar el acercamiento directo con las comunidades se debe realizar un proceso de reconocimiento del territorio. Esto se llevó a cabo en tres momentos:

Un primer momento consistió en la identificación del riesgo barrial a través de la caracterización sociodemográfica de los determinantes sociales de la salud, proceso que se realizó para la selección de los barrios sujeto de la investigación. Aquí se llevó a cabo un análisis del tipo y las condiciones de vivienda, estrato socioeconómico, género, estado civil, régimen de aseguramiento, escolaridad, condiciones de trabajo, hacinamiento, entre otros.

Para este caso, el equipo investigador obtuvo la información a través de la plataforma del Observatorio Social de la Dirección Territorial de Salud de Caldas, teniendo en cuenta que las herramientas, los software o las estrategias seleccionadas son elegidos de acuerdo al contexto local y nacional de cada investigación.

Posterior a la selección de los barrios se procedió con un segundo momento, la identificación de las zonas y límites geográficos, con el fin de obtener el mapa de base para la realización de la cartografía social con las comunidades. Para este proceso se contó con las herramientas de Google Maps y el referenciador geográfico del software Atlas.ti (Figura 3).

Además, la validación de algunos de los mapas pudo hacerse a través de las oficinas de planeación de los entes gubernamentales locales. Cabe aclarar que esto también puede realizarse con los entes departamentales o nacionales, según sea el caso.

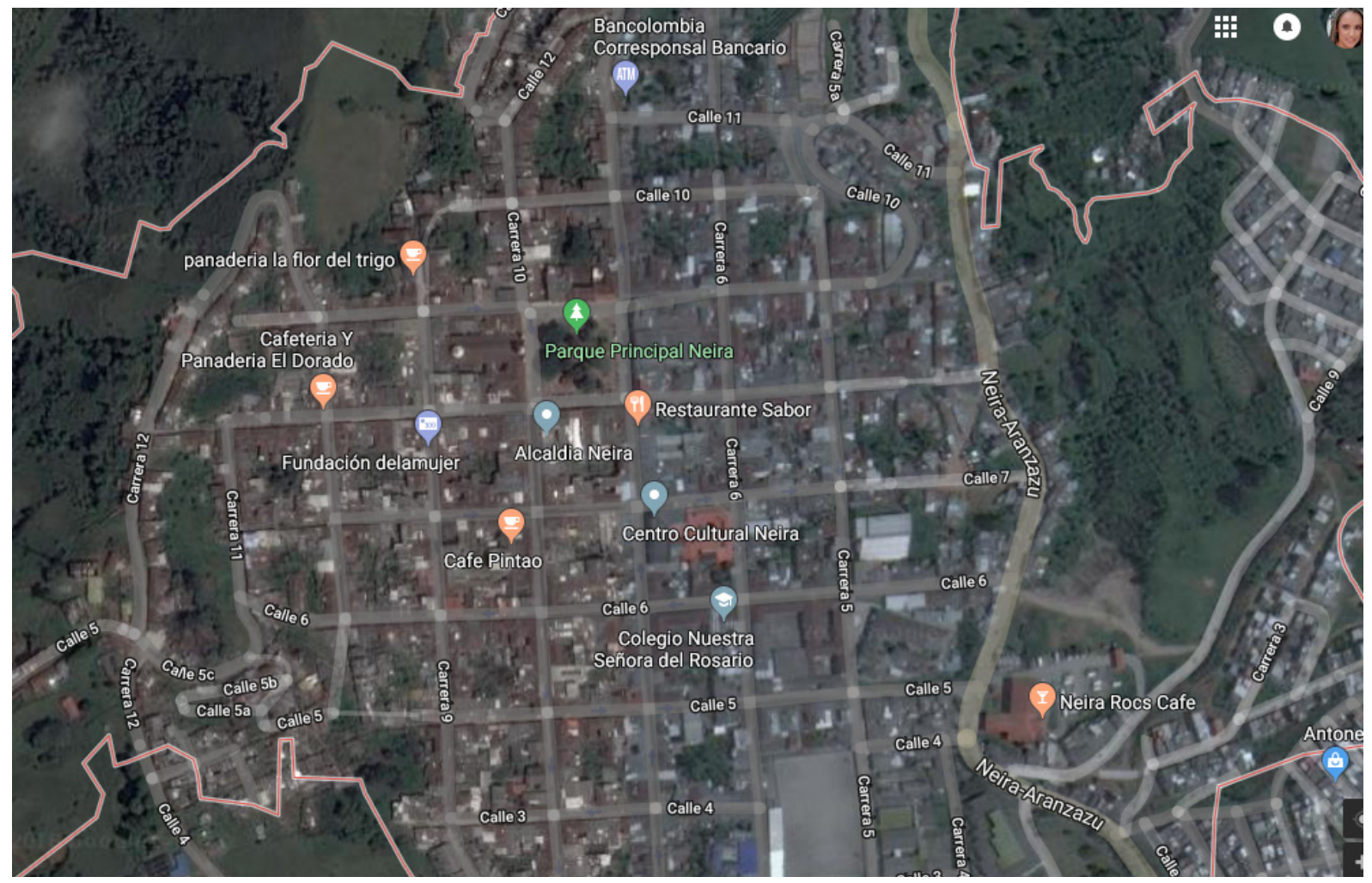

Figura 3. Ejemplo mapa georreferenciado para la construcción de ma. Fuente: Referenciador geográfico Atlas.ti. 
Finalmente, en el tercer momento, el equipo investigador realizó la primera visita, en donde, con el mapa impreso, llevó a cabo un recorrido por los barrios seleccionados para hacer un reconocimiento inicial de los límites barriales $y$, a su vez, reconocer de forma preliminar por observación no participante la población y los recursos del barrio.

\subsection{Acercamiento a la comunidad}

El ingreso a los territorios requirió de una reflexión por parte del equipo investigador sobre qué se haría y cómo se realizaría el acercamiento con la comunidad, teniendo presente, por un lado, que cada población cuenta con características diferentes, ya sean comportamentales, culturales, políticas o estructurales del barrio. Y, por el otro, que el objetivo de la investigación no debía ser solo la obtención de información, sino que además debía ser un proceso participativo, de corresponsabilidad y retroalimentación constante.

Con base en lo anterior, una de las estrategias utilizadas previo a iniciar la cartografía fue enmarcar el trabajo de las investigadoras en programas o actividades que ya se encontraban instauradas en la comunidad (huertas comunitarias, programas de responsabilidad social con la comunidad, por ejemplo, aquellas realizadas con el banco Davivienda, entre otros). De esta manera fue posible facilitar la inmersión al campo, focalizar grupos conformados y, a su vez, evitar saturar a la comunidad con nuevos procesos.

Para realizar un trabajo integral con la comunidad, en donde sus integrantes fueran sujetos activos de la investigación y no solo espectadores, fue fundamental la motivación en la participación, la creación de lazos de confianza y la empatía. Primero, a través de la inmersión activa del equipo investigador en las actividades que la población realizaba. Segundo, priorizando sus necesidades, intereses y gustos $y$, tercero, dinamizando los procesos con el compartir de alimentos y a través ejercicios recreativos y reflexivos. Todo esto ajustándonos a los tiempos de los participantes.

Además, en el marco de los encuentros se realizaron jornadas de integración social, tales como sancochada ${ }^{4}$, chocolatada y natillada, esta última como motivo de celebración del mes de diciembre y cierre del proceso cartográfico.

\subsection{Herramientas de indagación comunitaria}

Si bien es cierto que la cartografía social se realiza de manera articulada con la comunidad a través de un proceso emergente, para identificar los recursos del barrio y consignarlos en un mapa, fue necesario elaborar herramientas preliminares para guiar el proceso, tales como:
- Guía de preguntas orientadoras. Elaborada por el equipo investigador a partir de los objetivos trazados, la bibliografía consultada y la experiencia. Las preguntas que la constituían fueron abiertas, de modo que promovieran el diálogo con la comunidad de forma espontánea y de manera tal que se pudiera intentar profundizar sobre lo que se identificaba como activos comunitarios en salud en un proceso de coconstrucción continuo.

- Guía de cartografía social. No hay una única manera de construirla y llevarla a cabo. La decisión de cómo hacerla depende de las intenciones del equipo investigador. Es por ello que para orientar la guía se tuvieron en cuenta elementos centrales de la cartografía de sentidos, percepciones y territorios; cartografía de activos en salud; cartografía de paisajes y cartografía de recorridos urbanos.

- Unidad didáctica. La planeación de cómo se llevarían a cabo los encuentros con la comunidad fue de vital importancia para evitar retrasos en los tiempos de cada etapa de la investigación. Para este caso, la unidad didáctica se constituyó en una valiosa herramienta. En ella se definieron las fechas de los encuentros, la manera en que se motivaría la población, los objetivos, el contenido a desarrollar, las estrategias, la metodología, los recursos que se necesitarían y cómo se evaluaría cada encuentro, aunque fue susceptible de modificaciones.

\subsection{Proceso de mapeo comunitario}

Una vez realizadas las tres etapas previas se desencadenó el proceso de mapeo comunitario, el cual se describe a continuación:

\subsection{Encuentros comunitarios como espacio de reconstrucción de sus recursos}

Durante los primeros encuentros fue necesario dar a conocer a los participantes los objetivos, intenciones, motivos e intereses del trabajo que se pretendía realizar. Posteriormente, a partir del mapa obtenido con las herramientas geográficas y la generación de los lazos de confianza (de la primera etapa), se elaboró una reconstrucción manual del mapa.

Simultáneamente, con ayuda de las herramientas elaboradas, se indagó sobre los recursos físicos, económicos y culturales del barrio, de las asociaciones formales e informales, de las organizaciones y de los sujetos, donde se incluyeron sus saberes populares. La ubicación de cada recurso debía ser muy específica, para facilitar posteriormente la construcción del dispositivo gráfico (mapa). 


\subsubsection{Recorridos del territorio}

Dado que la identificación de los activos comunitarios se hizo a través de un ejercicio de reflexión y rememoración por parte de la comunidad, se consideró complementar el proceso cartográfico con un recorrido por los barrios, con la compañía de los participantes del proyecto y los líderes y lideresas comunitarios, de tal manera que con ellos se hiciera el reconocimiento final de los activos presentes en la localidad, además de construir la historia barrial inmersa en cada territorio.

Para esto, primero el equipo investigador acordó una fecha y un tiempo estimado para la realización de cada recorrido - la variación dependía de la extensión del lugar -.Y, segundo, se coordinó la logística en relación con la manera en que se realizarían el recorrido, los refrigerios y la hidratación.

Al finalizar, todos los participantes ubicaron los recursos faltantes en el borrador del mapa que se había ido trabajando.

\subsubsection{Construcción y validación del mapa}

Este proceso se llevó a cabo a través de las siguientes actividades:
- Realización de primer borrador de mapa. Con los insumos del reconocimiento del barrio y el acercamiento con la comunidad, se llevó a cabo la primera validación de los límites barriales y se inició la ubicación de los activos comunitarios identificados en los encuentros con los participantes (Figura 4). De este momento, llamó la atención que los primeros recursos ubicados fueron las viviendas y que la comunidad no se familiarizó con la iconografía entregada por el equipo investigador, por lo que fue necesario modificarla.

- Encuentro con líderes y lideresas para revisar y validar el mapa. Una vez realizada la validación inicial del mapa y el recorrido del barrio, se procedió con las correcciones pertinentes de los activos en salud en el mapa. Seguidamente se acordó un encuentro con los participantes y líderes y lideresas comunitarios para su última revisión, validación y reajuste.

- Socialización y entrega formal del mapa a la comunidad y a tomadores de decisiones. Para el cierre del proceso investigativo se convocó a una sesión conjunta a la comunidad y a los tomadores de decisiones (responsables políticos de la administración local), con el fin de socializar los resultados y realizar la entrega final del mapa de activos comunitarios en salud (Figura $5)$.

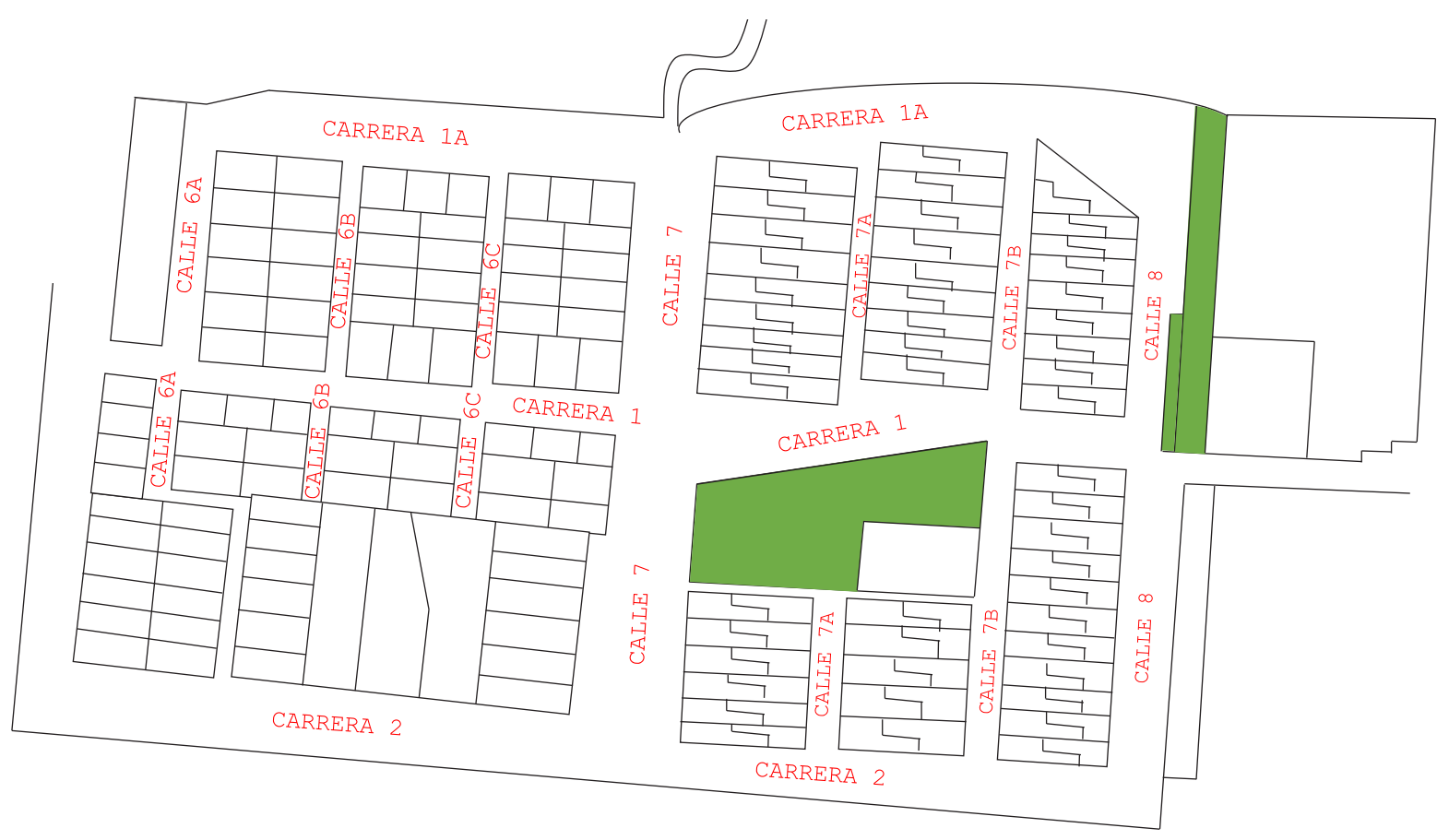

Figura 4. Borrador inicial del mapa de activos de unos de los barrios.

Fuente: Elaboración propia 


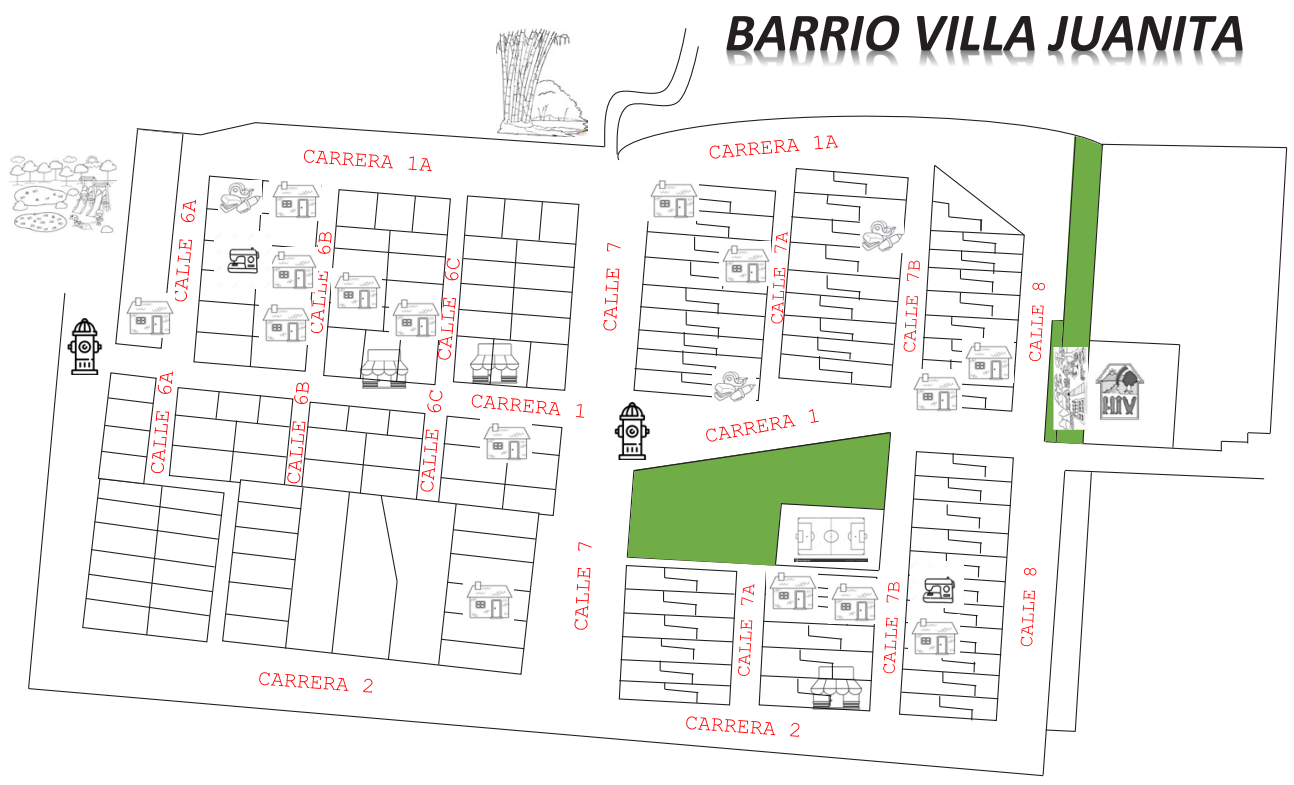

Figura 5. Mapa de activos final.

Fuente: Elaboración propia.

\subsection{Establecimiento de acuerdos sociales}

El proceso de mapeo comunitario fue diseñado para que los participantes pudieran darle continuidad. Se sugirió, por ejemplo, la cartografía socio-ambiental para construir la historia de los territorios, conflictos, actores, amenazas y el mapa de sueños (Soliz y Maldonado, 2006). En los últimos encuentros los participantes fueron conscientes de los activos y del deseo de realizar acuerdos sociales frente a la socialización de los activos con otras personas del barrio.

Estos acuerdos también permiten afianzar los procesos de gobernabilidad, defensa de la autonomía, permanencia y subsistencia para fortalecer los recursos existentes y buscar solución desde los recursos individuales, familiares y comunitarios con una visión participativa y transformadora, en función de mejorar su espacio de vida.

\section{Discusión de los resultados}

En la discusión se realiza el análisis de cómo se llevó a cabo el proceso de la cartografía social en este estudio. En este sentido, se destaca la importancia de la cartografía social y tres asuntos centrales: en primera instancia, el análisis del acceso al campo. En un segundo apartado, el mapeo desde activos comunitarios para visibilizar realidades positivas territoriales $y$, finalmente, los acuerdos sociales como proyecto de transformación barrial.

Según Braceras (20I2), el objetivo de la IAP es "transformar la realidad, pero con los propios afectados como protagonistas" (p. I5), lo que necesariamente requiere involucrar a la comunidad para que de manera activa identifique sus problemas, sus potencialidades y activos $y$, de igual manera, proponga acciones para generar cambios en su realidad. La cartografía social, considerada como un instrumento de IAP, de construcción y reflexión colectiva basada en las experiencias vividas y en el espacio construido por las comunidades, permite a través de herramientas como los mapas demarcar territorialmente, por parte de los actores interesados, aquellos elementos relevantes y que potencializan su desarrollo, bajo la premisa de que es necesario conocer el espacio para poder vivir en él (Oslender, 2017).

La cartografía social, como método, ayuda al investigador a familiarizarse con el trabajo de campo, potenciarlo y planearlo constantemente, utilizándolo simultáneamente como guía y método de reflexión constructiva -desde una mirada científica del territorio- (Osorio y Rojas, 20I I). Tal como lo describe Oslender (2017), constituye un ejercicio colectivo en que se exploran percepciones territoriales de las comunidades locales con el fin de plasmarlas en mapas que se instrumentalizan después como herramienta en la lucha por el territorio.

La cartografía social permite manejar la información de una manera dinámica y visual a través del mapeo, ayudando a identificar la percepción sobre la manera como los actores de la comunidad se relacionan con el entorno y las interacciones que se generan con el contexto y el territorio.

En algunos casos, es potenciadora de transformaciones comunitarias ya que en este acercamiento se pueden 
proponer ideas innovadoras en torno a cómo mejorar el territorio y la forma de vivir en él, según lo plantea López (20I8).

\section{I. Estrategias clave de acceso al campo para potenciar resultados positivos en la elaboración de la cartografía social}

Torrejón, Noboa y Oraisón (2017), en su investigación sobre la participación ciudadana en Latinoamérica, encontraron que para la elaboración participativa de cartografías socioterritoriales es necesario no solo contar con un espacio físico, sino también establecer redes de cooperación y lazos de confianza para lograr un verdadero proceso de articulación bajo el prisma de innovación social. De este modo, el acceso al campo fue clave en este estudio. Acá se destacan dos estrategias donde se anidó el estudio para generar empatía con la comunidad y que permitieron lograr resultados positivos.

Una de las estrategias utilizadas para esta investigación fue la vinculación del equipo en las actividades de huertas comunitarias realizadas en los barrios. Esto, tal como lo manifiestan Acuña, Guevara y Flores (2014), fue útil para lograr la creación de lazos de confianza, la participación efectiva de la comunidad, y para crear un compromiso individual y grupal para la participación. Sumado a que el proceso de socialización entre investigadores y participantes se produjo simultáneamente con el compartir de productos, experiencias y espacios de recreación, lo que en conjunto propició sobre la marcha una mejor convivencia y una mayor participación en las actividades comunes.

Otra de las estrategias utilizadas para el acceso a campo fue la participación del equipo investigador en las reuniones realizadas por los líderes y lideresas de los municipios, que en este caso se llevaban a cabo en el marco de un proceso de extensión social de círculos de la cultura, que como lo plantea Paulo Freire son:

Un lugar -junto a un árbol, en la sala de una
casa, en una fábrica, pero también una escuela-
donde un grupo de personas se reúne para
discutir sobre la práctica: su trabajo, la realidad
local y nacional, su vida familiar, etc. En el círculo
de cultura los grupos que se reúnen aprenden a
leer y a escribir, al mismo tiempo que aprenden a
"leer" (analizar y actuar) su práctica. Los círculos
de cultura son unidades de enseñanza. (Gadotti
y Torres, 200 I, p. 720)

Por lo anterior, las estrategias a desarrollar en la comunidad para generar confianza en el acceso a campo dependen en 148 se les otorgue, tal como lo plantean García et al. (20l6), Quienes resaltan que, en el desarrollo de las actividades comunitarias, los profesionales deben procurar que la participación de la comunidad (que históricamente ha sido relegada al papel de simple espectadora) sea activa y protagonista desde el comienzo, para que esto no se convierta en un obstáculo, sobre todo en aquellas comunidades que han sido sobre diagnosticadas, dado que esta es la fase que conlleva más tiempo y la cual es crucial para lograr determinado impacto.

\subsection{El mapeo de activos comunitarios en salud para visibilizar la realidad positiva territorial}

El mapeo de activos comunitarios en salud permite la construcción de la realidad territorial. Al respecto, autores como Diez et al. (2018) plantean que el análisis del territorio a través de metodologías participativas -en este caso la cartografía social- permite construir mapeos con contenidos sociales y datos cruzados levantados por los agentes comunitarios, lo que posibilita actuar con mayor eficacia ante situaciones relacionadas con el patrimonio de salud de las personas, familias y sus colectivos.

El mapeo de activos permite a la población incrementar la capacidad de trabajo conjunto (acción comunitaria en salud) y fortalecerse para conseguir cambios transformadores. Es así como, con la presente investigación, fue posible en el trabajo de campo trascender la visión de riesgo centrada en la identificación de necesidades y problemas de los barrios. Al respecto,Aviñó (2017) señala la importancia de dar paso a una visión que permita reconocer las fortalezas, recursos y oportunidades de mejora de las comunidades, es decir, que se favorezca la dinamización de las acciones comunitarias, el fortalecimiento de la capacidad de conexión entre los actores en pro de un mismo propósito y, finalmente, la construcción de un espacio creativo y activo para la toma de decisiones.

Cuando se visibilizan los aspectos positivos de una comunidad, superando una visión victimizante de pérdidas constantes, estigma, desesperanzas o riesgos -que para esta investigación fue el trabajo de mapeo de activos en salud-, se promueve el sentido de pertenencia de quienes participan del proceso, tal como lo señala Oliva (2015), pues manifiesta que con el mapeo de activos es posible desencadenar un mayor interés de la población, una conexión emocional entre todos los vecinos y una creencia compartida sobre lo bueno y lo mejor del lugar en el que viven, es decir, una mayor construcción de identidad barrial.

De esta manera, el mapeo de activos comunitarios en salud les permite a las personas y comunidades identificar $y$ 
reconocer que, a pesar de que aún existen necesidades y problemas, es posible y esencial valerse de aquellos recursos con los que cuentan para continuar con la construcción de su territorio, y así afrontar y superar algunos de los obstáculos sociales, económicos y políticos que generan desigualdades sociales y en salud (Morgan y Hernán, 2013).

\subsection{Los acuerdos sociales como proyecto de transformación barrial}

Los encuentros sociales planeados y con una intencionalidad, en este caso la elaboración de los mapas con participación comunitaria, confirman lo que plantea Wiesenfeld (201520l6), quien resalta cómo los encuentros crean espacios adecuados para el enriquecimiento de la comunidad, su desarrollo y el fomento de una participación comunitaria transformadora. En este sentido, estudios realizados resaltan cómo, mediante las experiencias de la cartografía participativa, se genera la valorización de lo comunitario y la potencial protección de bienes comunes con participación de todos los actores (Quiñónez, 20II).

Uno de los elementos más altamente valorados en la investigación está ligado al proceso motivador de trabajo colectivo, el reconocimiento mutuo, el hecho de darle voz a la comunidad y hacerla partícipe de un proceso que prepara a sus integrantes para cambios futuros -los sueños-. Los acuerdos sociales, que se generan en la fase final del estudio, se avizoran como un proyecto de transformación barrial, en tanto se convierten en la posibilidad de que la comunidad se dé cuenta de los recursos y potencial con el que cuenta, la percepción del mundo barrial como un espacio fértil para los procesos colaborativos, la generación de nuevas iniciativas, y se comprometa en mejorar su espacio de vida como una decisión autónoma (Aviñó, 2017).

De este modo, mapear no debe quedarse en el cumplimiento de compromisos adquiridos durante un ejercicio académico. Va más allá. La apuesta es la interpretación -conocimiento "en algo" del mundo a través de territorios concretos-cuya intención va orientada a la transformación de los micro contextos, lo que permite aportar a la justicia, la equidad y la solidaridad humana.

\subsection{Limitaciones}

Las autoras reconocen como limitaciones para la realización del presente trabajo, las siguientes:

- Los encuentros con la comunidad algunas veces pueden verse frustrados por diversos factores como falta de asistencia, estrategias poco efectivas de convocatoria, actividades programadas en los municipios, promesas que nos les fueron cumplidas, calamidades de los líderes y lideresas, distancias con el sitio de reunión, entre otros.

- La iconografía utilizada para el proceso cartográfico no siempre es comprendida por la comunidad, por lo que se deben hacer modificaciones correspondientes.

- Para la continuación de esta investigación, es importante vincular otras áreas disciplinares desde las ciencias sociales que permita comprender y problematizar el territorio como fenómeno complejo en relación con la salud pública.

\section{Conclusiones}

La validez de la cartografía social como método de investigación se evidenció en los resultados de este trabajo, ya que posibilitó la generación de conocimiento a partir de la aplicación del diseño teórico y la recontextualización que se realizó sobre los activos comunitarios como temática central, lo anterior abre horizontes para resignificar la importancia de su aplicación en salud.

La ruta de investigación que subyace al método aplicado y las etapas desarrolladas (conocimiento del territorio, acercamiento a la comunidad, herramientas de indagación comunitaria, proceso de mapeo comunitario y establecimiento de acuerdos sociales), sustentan la relevancia que para el trabajo comunitario y la salud tiene la aplicación del método de cartografía social.

La cartografía social permite el análisis de la situación de salud con el impulso de la participación colectiva, haciendo partícipes a los actores del proceso de generación de nuevo conocimiento para que a partir de este se formulen las políticas y planes de salud.

La cartografía social se puede considerar un medio para que las comunidades reflexionen, socialicen saberes $y$ prácticas y analicen su realidad, reconociendo aquellos elementos en los que se desarrolla su vida cotidiana. Por tanto, podría afirmarse que la cartografía social aporta para la elaboración de rutas de vida a nivel comunitario en el marco de la Atención Primaria en Salud.

El modelo de activos, expresado a través de la cartografía social, hace posible que la comunidad reconozca sus elementos territoriales y fortalezas personales, familiares y sociales para responder a las necesidades concretas en salud de una manera integral, al superar un enfoque centrado únicamente en el riesgo.

Se recomienda para futuras investigaciones realizar un reconocimiento detenido del territorio $y$ de las comunidades, con el fin de identificar los programas y proyectos desarrollados en este y convertirlos en 
potenciales puertas de entrada para el trabajo de campo de la cartografía social. Previo a la realización del mapeo colectivo, es necesaria la sensibilización y resignificación del concepto de "recursos comunitarios" en las comunidades.

\section{Conflicto de intereses}

Las autoras declaran no tener ningún conflicto de intereses.

\section{Notas}

I. Según Chapela (2008): “apoderar hace referencia a alguien que por sus propias razones y con sus propios medios $y$ fines, decide dotar a otra persona de algo que posee y quien es dotado no posee, estableciendo así una relación en donde una parte es activa e independiente y la otra pasiva y dependiente. Esta es frecuentemente la interpretación de empowerment que se hace en los discursos oficiales que perpetúan la relación de dominio sobre la población, cosa contraria a la intención de apoderar en donde el sujeto se dota y recupera lo que es y era suyo en primera instancia".

2. Según el Observatorio Social de Atención Primaria Social de la Dirección Territorial de Salud de Caldas (https:// saluddecaldas.gov.co/observatorio-social/).

3. Categorías: recursos de los sujetos, recursos de las asociaciones formales, recursos de las asociaciones informales, recursos físicos del área, recursos económicos, recursos culturales, recursos de las organizaciones, metodología y saberes populares.

4. Alimento popular realizado con base en caldo, tubérculos y carne.

\section{Referencias bibliográficas}

I. ACUÑA, Iraima; GUEVARA, Harold y FLORES, Mitzy. El empoderamiento como estrategia de salud para las comunidades. En: Revista Cubana de Salud Pública. 20I4. vol. 40, no. 3, p. 353-36I. Disponible en: https://www.medigraphic.com/pdfs/revcubsalpub/csp20|4/csp|43e.pdf

2. ANTONOVSKY, Aaron. Unraveling the Mystery of Health. How People Manage Stress and Stay Well. San Francisco:Jossey-Bass, 1987a.

3. ANTONOVSKY, Aaron. The salutogenic perspective: toward a new view of health and illness. In: Advances. 1987b. vol. 4, no. I, p. 47-55. Disponible en: http://psycnet.apa.org/psycinfo//988-2016I-00|

4. AVIÑÓ, Juan. Mapeo de activos en salud en dos barrios vulnerables y su dinamización en una intervención comunitaria participativa. Tesis doctoral Enfermería. España: Universidad de Valencia, 2017. 344 p. Disponible en: http://roderic.uv.es/handle//0550/60804

5. BARRAGÁN, Andrea. Cartografía social: lenguaje creativo para la investigación cualitativa. En: Revista Sociedad y Economía. 2019. vol. 36, p. 139-159. https://doi.org//0.25100/sye.v0i36.7457

6. BARRAGÁN, Diego Fernando. Cartografía social pedagógica: entre teoría y metodología. En: Revista Colombiana de Educación. Enerojunio 2016. no. 70, p. 247-285. Disponible en: http://www.scielo.org. co/pdf/rcde/n70/n70al 2.pdf

7. BARRAGÁN, Diego Fernando y AMADOR, Juan Carlos. La cartografía social-pedagógica: Una oportunidad para producir conocimiento y repensar la educación. En: Itinerario Educativo. Diciembre 20I4. vol. 28, no. 64, p. |27-|4|. Disponible en: http://revistas.usbbog.edu.co/ index.php//tinerario/article/view//422

8. BRACERAS, Iratxe. Cartografía participativa: herramienta de empoderamiento y participación por el derecho al territorio. Trabajo de grado Máster Desarrollo y Cooperación Internacional. España: Universidad del País Vasco - Instituto de Estudios sobre Desarrollo y Cooperación Internacional, 2012.55 p.

9. CALDERON, Carlos. Criterios de calidad en la Investigación Cualitativa en Salud (ICS): apuntes para un debate necesario. En: Revista Española de Salud Pública. Septiembre 2002. vol. 76, no. 5, p. 473-482.

10. CHAPELA, María del Consuelo. Promoción de la salud. Un instrumento del poder y una alternativa emancipatoria. En: University of New México, 2008. Disponible en: http://www.famp.es/racs/ ObsSalud/documentos/Promocion_de_la_Salud.pdf

II. COFIÑO, Rafael; AVIÑÓ, Dory; BENEDÉ, Carmen Belén; BOTELLO, Blanca; CUBILLO, Jara; MORGAN, Antony; PAREDES, Joan Josep y HERNÁN, Mariano. Promoción de la salud basada en activos: ¿cómo trabajar con esta perspectiva en intervenciones locales? En: Gaceta Sanitaria. Noviembre 2016. vol. 30, no. I, p. 93-98. https://doi. org/10.1016/j.gaceta.2016.06.004

12. DE LA REVILLA, Luis. Conceptos e instrumentos de la atención familiar. Barcelona: Doyma, 1993.

13. DIEZ, Juan Manuel; ROCHA, Eduardo; MUNSBERG, Glauco; PEIXOTO, Jessica Helena; NEUTZLING, Aline; JAIME, Sergio Fernando y SCHULER, Leonardo Javier. Desarrollo de un sistema georreferenciado para la gestión, movilidad y monitoreo de atención primaria de la salud comunitaria. En: Salud Colectiva. Abril 2018. vol. I4, no. I, p. I2I-137. https://doi.org//0.18294/sc.2018.1210

14. GADOTTI, Moacir y TORRES, Carlos Alberto. comps. Paulo Freire: una biobibliografía. México D.F.: Siglo XXI, 200I.

I5 GANTER, Rodrigo; SANDOVAL, Daniel; GARCÍA, Daniela y DE LA FUENTE, Helen. Topofilia y cartografías participativas en el sur de Chile. La experiencia comparada en las ciudades de TemucoPadre las Casas, Valdivia y el Gran Concepción. En: Prisma Social. 2015. no. 15, p. 440-49I. Disponible en: http://www.redalyc.org/ pdf/3537/353744533013.pdf

16. GARCÍA, Ana M.; BOIX, Pere; BENAVIDES, Fernando G.; GADEA, Rafael; RODRIGO, Fernando y SERRA, Consol. Participación para mejorar las condiciones de trabajo: evidencias y experiencias. En: Gaceta Sanitaria. Noviembre 2016. vol. 30, no. I, p. 87-92. https://doi. org/10.1016/i.gaceta.2016.03.006

17. IGLESIAS, José Antonio; CASADO, Inés; POSTIGO, Salvador; BÁRCENA, Carmen; GALÁN, Isabel y DEL VALLE, M. José. La salud comunitaria basada en activos para la salud. Un modelo que no cala en el quehacer cotidiano. En: Revista Enfermería CyL. Enero 2018. vol. I0, no. I, p. 17-22. Disponible en: http://www.revistaenfermeriacyl. com/index.php/revistaenfermeriacyl/article/viewFile/207//81

18. LÓPEZ, César Enrique. La cartografía social como herramienta educativa. En: Revista Scientific. Noviembre 2018. vol. 3, no. 10, p. 232-247. https://doi.org//0.29394/Scientific.issn.25422987.2018.3.10.12.232-247

19. MONTES, César A.;JAIMES, Yanela P.;AYALA, Ángela P.; RODRÍGUEZ, Yohandris; LLANOS,Andrés;ASCANIO,Yessica; BLANCO, Kelly Paola; VILLA, Fidelina, et al. Determinantes sociales en salud: construcción de conocimiento a través de la cartografía social. En: Revista Signos Fónicos. Octubre 2015 / marzo 2016. vol. I, no. 3, p. I-74. http:// revistas.unipamplona.edu.co/ojs_viceinves/index.php/CDH/article/ view/ $/ 888 / 908$ 
20. MORGAN, Antony y HERNÁN, Mariano. Editorial. Promoción de la salud y del bienestar a través del modelo de activos. En: Rev Esp Sanid Penit. 20I3. no. 15, p. 78-86. Disponible en: http://scielo.isciii.es/pdf/ sanipe/v15n3/es_0I_editorial.pdf

21. MORGAN, Antony y ZIGLIO, Erio. Revitalising the evidence base for public health: an assets model. En: Promoción y educación. Junio 2007. vol. I4, no. 2, p. 17-22. https://doi.org/I0.1177\%2FI0253823070I4002 $\underline{0701 x}$

22. MORGAN, Antony; DAVIES, Maggie y ZIGLIO, Erio. eds. International health and development: investing in assets of individuals, communities and organizations. New York: Springer-Verlag, 2010.

23. OLIVA, Alfredo. Los activos para la promoción del desarrollo positivo adolescente. En: Metamorfosis. Diciembre 2015. no. 3, p. 32-47. Disponible en: https://dialnet.unirioja.es/servlet/ articulo?codigo $=6163218$

24. ORGANIZACIÓN MUNDIAL DE LA SALUD. Conferencia Internacional sobre Atención Primaria de Salud - Declaración de Alma-Ata. Ira Ed. Suiza: Oficina de Publicaciones, Organización Mundial de la Salud, Fondo de las Naciones Unidas para la Infancia, 1978.

25. ORGANIZACIÓN MUNDIAL DE LA SALUD. Carta de Ottawa para la Promoción de la Salud. Estados Unidos: Oficina de prensa OMS, 1986.

26. OSLENDER, Ulrich. Ontología relacional y cartografía social: ¿hacia un contra-mapeo emancipador, o ilusión contra-hegemónica? En:Tabula Rasa. Enero-febrero 2017. no. 26, p. 247-262. http://dx.doi. org/l0.25058/20II2742.196

27. OSORIO, Henry y ROJAS, Edilsa. La cartografía como medio investigativo y pedagógico. En: Dearq. Diciembre 20l l. vol. 9, p. 3047. Disponible en: https://revistas.uniandes.edu.co/doi/pdf//0.18389/ dearq9.2011.05

28. PÁJARO, David y TELLO, Enriqueta. Fundamentos epistemológicos para la cartografía participativa. En: Revista Etnoecológica. Abril 2014. vol. I0, no. I, p. I-20. Disponible en: https://www.researchgate.net/ publication/296665874_Fundamentos_epistemologicos_para_la cartografia_participativa

29. QUIÑÓNEZ, María Carmela. Una experiencia de cartografía social en la zona de bajamar -Isla de Cascajal Buenaventura-. En: Entramado. Julio-diciembre 20II. vol. 7, no. 2, p. I56-I7I. Disponible en: http:// www.scielo.org.co/pdf/entra/v7n2/v7n2a 10.pdf

30. SCALES, Peter C. Reducing risks and building developmental assets: essential actions for promoting adolescent health. En:The Journal of school health. Octubre 1999. vol. 69, no. 3, p. II3-I19. https://doi. org/10.1111//..1746-1561.1999.tb07219.x

3I. SOLIZ, Fernanda y MALDONADO, Adolfo. Guía de metodologías comunitarias participativas. Bogotá: Clínica ambiental, 2006. 54 p. Disponible en: https://mx.123dok.com/document/4yr69x8ysoliz-fernanda-y-adolfo-maldonado-2006-guia-de-metodologiascomunitarias-participativas-primera-edicion-clinica-ambiental-bogota-colombia.html

32. TORREjÓN, Eryka; NOBOA, Alejandro y ORAISÓN, Mercedes. Dossier "La participación en América Latina: Formas emergentes y cristalizadas de politicidad y prácticas ciudadanas": Introducción. En: De Prácticas y Discursos. Enero-julio 2017.vol.6,no.7,p.2-9.Disponible en: http://ppct.caicyt.gov.ar/index.php/depracticasydiscursos/article/ view/10438

33. URIBE, Claudia Patricia; DONOSO, Diana y RAMÍREZ, Alfredo. De la cartografía social a la comprensión de los contextos socioeducativos. En: Aletheia. Noviembre 2017. vol. 9, no. 2, p. 74-93. Disponible en: http://www.scielo.org.co/scielo.php?script=sci_abstract\&pid $=\$ 2145-$ $03662017000200074 \& \operatorname{lng}=\mathrm{e} \& \mathrm{nrm}=\mathrm{iso} \& \mathrm{tlng}=\mathrm{es}$
34. WIESENFELD, Esther. Las intermitencias de la participación comunitaria: Ambigüedades y retos para su investigación y práctica. En: Psicología, Conocimiento y Sociedad. Noviembre 2015/abril 2016. vol. 5, no. 2, p. 335-387. Disponible en: http://www.scielo.edu.uy/pdf/ $\mathrm{pcs} / \mathrm{v} 5 \mathrm{n} 2 / \mathrm{v} 5 \mathrm{n} 2 \mathrm{a} / 4 . \mathrm{pdf}$

35. YEPES, Carlos Enrique y MARÍN, Yuli Andrea. Desafíos del análisis de la situación de salud en Colombia. En: Biomédica. Junio 2018. vol. 38, no. 2, p. 162-172. https://doi.org//0.7705/biomedica.v38i0.3594 